\title{
Sporadic Hemiplegic Migraine with SCN1A Gene Mutation-A Case Report
}

\author{
Mukesh Dube, ${ }^{1}$ Akshay Navalkishor Lakhotia, ${ }^{1}$ Vaibhav Yadav ${ }^{2}$ and Rahul Jain ${ }^{1}$ \\ 1. Department of Neurology, Sri Aurobindo Medical College and PG Institute, Indore, India; 2. Department of Medicine, Sri Aurobindo Medical College \\ and PG Institute, Indore, India
}

DOI: https://doi.org/10.17925/USN.2018.14.2.108

S poradic hemiplegic migraine (SHM) is a subtype of hemiplegic migraine, characterized by episodes of migraine with a reversible motor aura, without a positive family history, and is a mimicker of an atypical severe form of migraine, stroke, epilepsy, multiple sclerosis, metabolic disorders, or conversion disorder. Case presentation: We present the case of a young 28-year-old female, who had a history of recurrent reversible attacks of headache with sensory aura accompanied with left hemiparesis for the past 5 years, with no positive family history of similar symptoms. The work-up ruled out differential diagnoses and genetic work-up found a novel SCN1A gene missense variation in exon 26 (c.4855A>G; p.Met1619Val) in a case of SHM. She was discharged on flunarizine for prophylaxis. Conclusions: We describe, for the first time, a case of SHM with a mutation in the SCN1A gene.

\section{Keywords}

Sporadic hemiplegic migraine, SCN1A gene, CACNA1A gene, ATP1A2 gene

Disclosures: Mukesh Dube, Akshay Navalkishor Lakhotia, Vaibhav Yadav, and Rahul Jain have nothing to disclose in relation to this article.

Review Process: Double-blind peer review.

Compliance with Ethics: All procedures were followed in accordance with the responsible committee on human experimentation and with the Helsinki Declaration of 1975 and subsequent revisions, and informed consent was received from the patient involved in this case study.

Authorship: The named authors meet the International Committee of Medical Journal Editors (ICMJE) criteria for authorship of this manuscript, take responsibility for the integrity of the work as a whole, and have given final approval for the version to be published.

Open Access: This article is published under the Creative Commons Attribution Non-commercial License, which permits any non-commercial use, distribution, adaptation, and reproduction provided the original authors and source are given appropriate credit. (C) The Authors 2018.

Received: January 16, 2018

Accepted: July 20, 2018

Citation: US Neurology. 2018;14(2):108-10

Corresponding Author: Mukesh Dube,

4th Floor, Department of Neurology, Sri Aurobindo

Medical College and PG Institute, Sanwer Road, Indore,

Madhya Pradesh, India.E: dube_mukesh@yahoo.com

Support: No funding was received in

the publication of this article.
Hemiplegic migraine (HM) has been classified by the International Headache Society under the heading of migraine with aura (1.2). HM (1.2.3) occurs in two forms: familial (FHM 1.2.3.1) and sporadic (SHM 1.2.3.2). ${ }^{1}$ Migraine attacks are associated with a gradual progression of motor weakness/hemiparesis during the aura phase and where no first- or second-degree relative (parent, sibling, or child) has had identical attacks. The complex aura symptoms of fever, lethargy, dysphasia, confusion, hemiparesis, hemisensory symptoms, hemianopia, and scintillating scotoma are associated with the phenotype of SHM, akin to stroke. In lieu of no diagnostic marker, the diagnosis of SHM in most cases is essentially one of exclusion.

\section{Case presentation}

A 28-year-old, married, Hindu Asian, right-handed female presented with a history of episodic attacks of headache, along with transient weakness of the left half of her body occurring twice a week for the past 5 years. She had bifrontal, throbbing headache associated with nausea, photophobia, and phonophobia, persisting for 4-6 hours. Each episode was associated with a tingling sensation starting from the left upper limb and involving the left lower limb as well, followed by weakness in a similar distribution. The progression of sensory symptoms took 10-20 minutes to reach its peak and later subsided in the next 30-45 minutes, to be followed by left hemiparesis which, along with the headache, lasted for 4-6 hours with complete recovery. Some episodes were associated with transient diminution of vision and vertigo. No scotomas or flashes of light were experienced.

There was no history of syncope, altered sensorium, confusion, language abnormalities, abnormal body movements during or after the episodes, myoclonus, muscle pains or muscle weakness, connective tissue disorder, coagulation disorders, diabetes mellitus, cardiac disease, abortions, smoking, or illicit drug abuse. There was no history of birth insult. None of the first- and second-degree relatives had similar complaints (Figure 1). Detailed general examination revealed pallor, and neurological examination was normal except for power in the left upper and lower limbs being Medical Research Council (MRC) grade IV/V. In the given clinical context, possibilities considered were: 1) anterior circulation transient ischemic attack; 2) complex partial seizure without secondary generalization with postictal Todd's palsy; 3) migraine with aura subtypes: (a) SHM or FHM, (b) basilar-type migraine, or (c) migrainous infarction.

Hematological profile showed microcytic hypochromic anemia, with mildly raised erythrocyte sedimentation rate, and normal hepatic and renal functions. Electrocardiogram showed sinus rhythm. Normal serum lactate levels with the forearm exercise test showed no evidence of defective oxygen extraction. Cerebrospinal fluid (CSF) was acellular, with normal proteins 


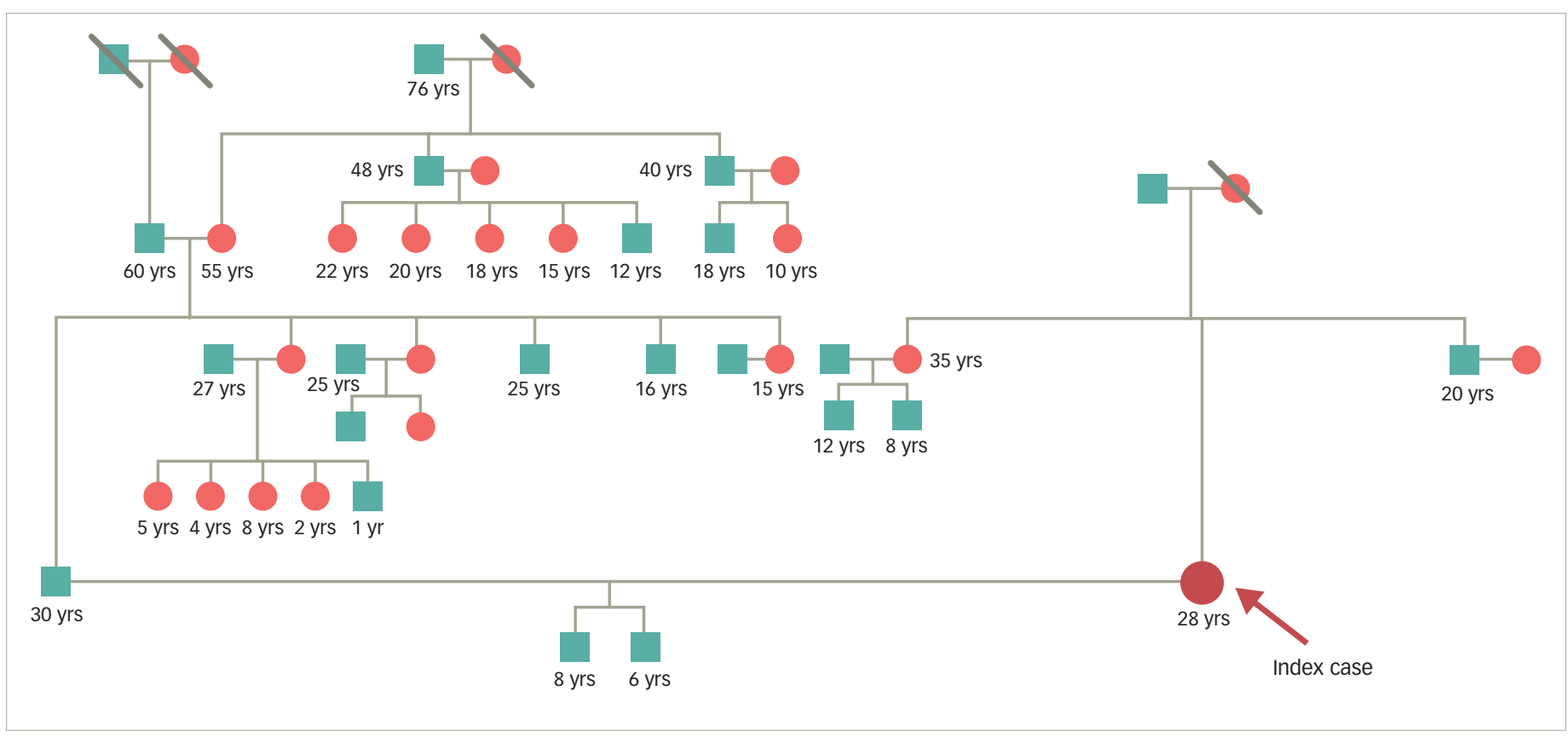

yrs $=$ years

and sugar. She had a normal magnetic resonance image (MRI) brain scan with normal magnetic resonance angiography of brain and cervical arteries. Extended 6-hour electroencephalogram was normal. The diagnosis of SHM was thus made.

In addition, molecular genetic testing was done, which displayed a missense variation in exon 26 of the SCN1A gene that resulted in the amino-acid substitution of valine for methionine at codon 1619. The Met 1619Val variant had a minor allele frequency of $0.06 \%$ in both the 1,000 genomes and EXAC databases. The in silico predictions of the variant were possibly damaged by Polyphen-2 (HumDiv), likelihood ratio test and mutation transfer 2 . The reference codon was conserved across the species. Based on this, SCN1A variation was classified as a likely pathogenic variant. The exon 26 of SCN1A was amplified by polymerase chain reaction and the product was sequenced using the Sanger method. The same variation was detected in a heterozygous condition by Sanger sequencing. Due to the unwillingness of the patient's relatives and financial constraints, genotype-phenotype segregation was not carried out.

She was treated with flunarizine $10 \mathrm{mg}$ once a day as migraine prophylaxis, and naproxen for acute management.

\section{Conclusion}

Our patient had a history of throbbing diffuse headaches accompanied by sensory symptoms and associated hemiparesis. There were no migrainous headache events without accompanying aura. There was no family history of such episodes. Transient ischemic attack and epilepsy are the common mimickers, but the gradual progression of symptoms and severe and prolonged postictal headache phase is uncommon. In this case, the work-up was not suggestive of the possibility of a stroke or epilepsy. CADASIL (cerebral autosomal dominant arteriopathy with subcortical infarcts and leukoencephalopathy) and amyloid angiopathy have been associated with $\mathrm{HM}$; the lack of history of permanent neurological deficits, dementia, family history, and normal brain MRI pointed against these factors. ${ }^{2}$ MELAS (mitochondrial encephalomyopathy, lactic acidosis, and stroke) is characterized by migrainous headaches and stroke-like focal deficits, biochemically associated with increased lactate in serum and CSF during the attack. MRI of the brain often reveals posterior cerebral cortex-predominant T2 and FLAIR hyperintense lesions. ${ }^{3}$ Normal serum lactate, normal forearm exercise, and normal brain imaging tests contradicted the diagnosis of MELAS. Multiple attacks of migraine-like headaches are seen in the syndrome of headache transient neurologic deficits and CSF pleocytosis (HaNDL), which is associated with weakness, sensory and visual auras with confusion, and on neurological examination meningismus lasting minutes to hours. The CSF shows lymphocytic pleocytosis with normal CSF protein and glucose with normal brain imaging. Our patient had no evidence of meningitis as well as no CSF abnormality. Although there was the presence of visual blurring in a few episodes, the presence of hemiplegia argues against the diagnosis of basilar migraine, as motor weakness is not part of the aura spectrum accompanying it. In migrainous infarction, on the side of the aura origin, large artery territory stroke is the hallmark. Prolonged hemiparesis associated with MRI evidence of infarction confirms the diagnosis. In the present case, normal brain MRI ruled out migrainous infarction. As an entity, prolonged aura without infarction, the prolonged aura with symptoms and signs lasts for more than 2 weeks without any radiologic evidence of infarction, while in our case the aura was shortlived. Our patient best fits into the diagnosis of migraine with aura and its subtype of $\mathrm{SHM}^{3,4}$ and fulfils the criteria.

Most patients with SHM have 'typical' aura symptoms (visual, sensory, and/or aphasic) associated with motor weakness during the acute attack..$^{3-5}$ The headache can be bilateral and precede the aura, with higher frequency of sensory, aphasic auras. The duration of SHM aura is longer compared with a typical migraine with aura. ${ }^{4}$ The aura of SHM comprising acute 
hemiplegic attack is always associated with severe headache, while the aura of typical migraine may occur without headache. ${ }^{4}$ Minor head trauma can trigger $\mathrm{HM}^{,}{ }^{5}$ whereas minor head trauma is not a triggering factor of classical migraine with aura. In SHM, pathogenetic mutations in CACNA1A and ATP1A2 gene loci have been reported. ${ }^{\circ}$ No SCN1A mutation has been yet found.

We report, for the first time, a SCN1A gene missense variation in exon 26 (c.4855A $>$ G; p.Met1619Val) in a case of SHM. The association is suspicious, but needs to be proven as causal. The SCN1A gene (MIM\#182389), codes for the pore-forming voltage-gated $\mathrm{Na}^{+}$channel alpha subunit $\mathrm{Na}_{\mathrm{v}} 1.1$, and is located on chromosome 2q24 consisting of 26 coding exons. The $\mathrm{Na}_{v} 1.1$ protein has four domains (I-IV), each containing six transmembrane segments (S1-S6). The missense mutations take place in the pore region without any domain preference. In FHM cases the location of missense mutations has been observed at linkers (excluding S5 and S6), voltage sensor S4 and pore (S5, S6 + linker). ${ }^{8}$ Novel SCN1A missense mutation p.lle1498Met and p.Phe1661Leu, and heterozygous T1174S missense mutation of NaV1.1 have been identified in families with FHM. T1174S induces divergent functional effects that can be consistent with both a gain and a loss of function of $\mathrm{Na}_{\mathrm{v}}$ 1.1.? L263V exhibited gain-of-function features, including delayed entry into, as well as accelerated recovery from, fast inactivation; depolarizing shifts in the steady-state voltage dependence of fast and slow inactivation; increased persistent current; and delayed entry into slow inactivation. Notably, the two mutations (Q1489K and L1649Q) exhibited partial or complete loss of function. ${ }^{10}$

The hypothesis we put forward is that the cortical spreading wave of neuronal and glial depolarization across the cerebral cortex, known as cortical depression, followed by long-lasting suppression of neuronal activity, could be due to excessive firing of neurons expressing mutant $\mathrm{Na}_{\mathrm{v}} 1.1$. The Met1619Val missense mutation, causing gain or loss of function, can facilitate initiation and propagation of cortical-spreading depression. The repetitive firing of the channel might enhance the release of the excitatory neurotransmitter glutamate, a critical element in cortical-spreading depression. ${ }^{11}$

The management of HM is empirical, and nasal administration of ketamine for acute management of aura and intravenous verapamil for acute headache is used. The use of triptans in HM is still being debated. These drugs are historically contraindicated for the fear of their vasoconstrictor properties that can worsen the aura. ${ }^{2}$ However, in a retrospective study by Artto et al., triptans were indicated to be safe and effective for the treatment of the headache in attacks. ${ }^{12}$ Case reports of effective treatment with flunarizine, naloxane, verapamil, and acetazolamide form the basis of prophylactic treatment and were found to have beneficial effects in some patients with $\mathrm{FHM}^{2}{ }^{2}$
1. Headache Classification Committee of the International Headache Society (IHS). The International Classification of Headache Disorders, 3rd edition (beta version). Cephalalgia. 2013;33:629-808.

2. Russell MB. Management of sporadic and familial hemiplegic migraine. Exp Rev Neurotherapeut. 2010;10:381-7.

3. Bhatia R, Desai $\mathrm{S}$, Tripathi $\mathrm{M}$, et al. Sporadic hemiplegic migraine: report of a case with clinical and radiological features. $J$ Head Pain. 2008;9:385-8.

4. Russell MB, Ducros A. Sporadic and familial hemiplegic migraine: pathophysiological mechanisms, clinical characteristics, diagnosis, and management Lancet Neurol. 2011:10:457-70.
5. Saleh C, Pierquin G, Beyenburg S. Hemiplegic migraine presenting with prolonged somnolence: a case report. Case Rep Neurol. 2016;8:204-10.

6. Thomsen $L L$, Oestergaard E, Bjornsson A, et al. Screen for CACNA1A and ATP1A2 mutations in sporadic hemiplegic migraine patients. Cephalalgia. 2008:28:914-21.

7. Cestèle $S$, Labate A, Rusconi R, et al. Divergent effects of the T1174S SCN1A mutation associated with seizures and hemiplegic migraine. Epilepsia. 2013;54:927-35

8. Claes LR, Deprez L, Suls A, et al. The SCN1A variant database: a novel research and diagnostic tool. Human Mut. 2009;30: E904-20.
9. Weller CM, Pelzer N, de Vries B, et al. Two novel SCN1A mutations identified in families with familial hemiplegic migraine. Cephalalgia. 2014;3413:1062-9.

10. Kahlig KM, Rhodes TH, Pusch M, et al. Divergent sodium channel defects in familial hemiplegic migraine. Proc Nat Acad Sci. 2008:105:9799-804.

11. Dichgans $M$, Freilinger $T$, Eckstein $G$, et al. Mutation in the neuronal voltage-gated sodium channel SCN1A in familial hemiplegic migraine. Lancet. 2005;366:371-7.

12. Artto V, Nissilä M, Wessman $M$, et al. Treatment of hemiplegic migraine with triptans. Euro J Neurol. 2007;14:1053-6. 